RESEARCH PAPER

Available Online at www.ijarcs.info

\title{
COGNITIVE RADIO: CONCEPTS, SPECTRUM SENSING AND ITS STANDARDS
}

\author{
Shewangi \\ Research scholar \\ IT dept. UIET, Panjab university \\ Chandigarh, India
}

Roopali Garg

Assistant professor

IT dept. UIET, Panjab university

Chandigarh, India

\begin{abstract}
Cognitive radio is consider to be outlook of wireless communication system as it is responsible for many operations like using spectrum opportunistically, sensing the environment and its internal state. In this paper a brief overview of SDR or reconfigurable radio is discussed. Spectrum sensing is used to adapt, learn and aware of the environment. This problem is one of the most challenging issues in cognitive radio system. In this paper different sensing issues is presented. There are many standards that are used in wireless communications. Wireless technologies that need some sort of spectrum sensing for variation or for dynamic frequency access (DFA) are discussed.SDR is used to change the operation of the terminals. The cognitive radio is centrally a SDR which already knows the environment, state, position and therefore adjusts its operations according to the desired objectives. Recently many wireless standards started adopting cognitive features all of them are discussed in detail.
\end{abstract}

Keywords: Spectrum Sensing, Standards, SDR, Cooperative Sensing, Bluetooth

\section{INTRODUCTION}

In wireless communication most of the radio spectrum is underutilized thus result in low transmission rate and heavy traffic of network. So to overcome this issue, cognitive radio was introduced in 1991 by Joseph mittola.In CR there are two types of user, one is primary user which are licensed and another is secondary user which are unlicensed.CR has distinctive feature that it can share its spectrum with unlicensed spectrum when not in use. This technique is known as Dynamic spectrum Access.CR has potential to use spectrum efficiently. As shown in figure 1, spectrum holes are the portion of the spectrum which is unused. If this band is additionally used by licensed user the CR moves to other spectrum hole or remain in same band, changing its transmission in power level to avoid interference.

Spectrum management selects best available channel and it is divided into four steps: (a) spectrum sensing is used to sense the spectrums whether it is idle or not. (b) Spectrum decision decides for the finest selection of spectrum hole to transmit The data (c) Spectrum mobility is the capability to decide whether to vacant the channel or not. (d) Spectrum sharing is capability to share the spectrum with unlicensed users to increase the efficiency of spectrum utilization. [1] This method of sharing is called dynamic spectrum access. Among all the components spectrum sensing is the main and important because all the operations in CR depend on it. Sensing is used for measuring various aspects like measuring the radio frequency energy, types of signals occupied by the channel and spectral content. A number of methods are proposed to identifying the presence of signal which is discussed later in the paper. [2] [3]

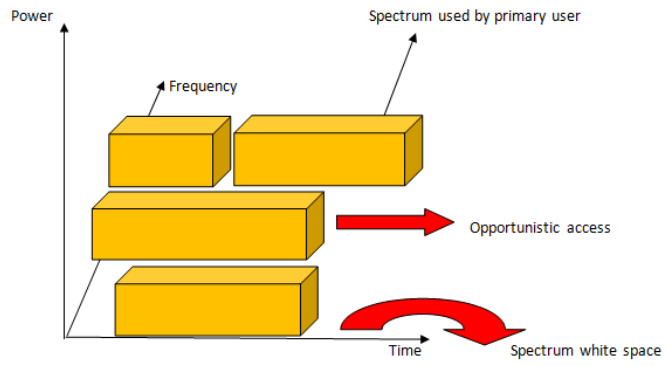

Fig 1: Spectrum white space model

1. Frequency: presence of frequency bands that can be split into smaller bands for proper usage for secondary users

2. Time: presence of a frequency band at a particular time

3. Geographical Space: presence of frequency bands in a particular geographical area

4. Code: presence of interfering simultaneous transmission based on various coding schemes

\section{A. Hardware Requirements}

In cognitive radios, sampling technique calls for high sampling rate, high resolution A/D convertors with huge dynamic range and high speed signal processor. The huge bandwidth requires additional requirements on the RF (Radio Frequencies) elements for e.g. power amplifier and antennas. These elements must be able to operate on wide range of frequencies.Additionaly for performing computational of signal high speed FGP or DSP is required with low delay. [4]

\section{B. Hidden primary user problem}

It is similar to hidden node problem in CSMA (Carrier Sense Multiple Accessing).In this problem the PU does not properly detected as CR device causes unwanted disturbance to the PU as a result, the multiple copies of PU arises and original PU becomes hidden. This problem can be caused by many factors like shadowing, multipath fading etc. So cooperative sensing arises to be tempting solution to enhance the performance of sensing and remove hidden user problem. 


\section{SDR}

A CR can also be SDR that is used for sensing, tracking changes and take action upon its finding. Software Defined Radio is used to provide supple radio functionality by avoiding the use of fixed components and analog circuits. Radio in which some or all of the physical layer functions are Software Defined

Figure 2 shows an SDR transceiver that can be reconfigured via a control bus supplying the processing units with the constrain which describe the desired standard. Such a configuration, called a parameter-controlled $(\mathrm{PaC}) \mathrm{SDR}$, guarantees that the transmission can be changed immediately if necessary (e.g., for interstandard handover).

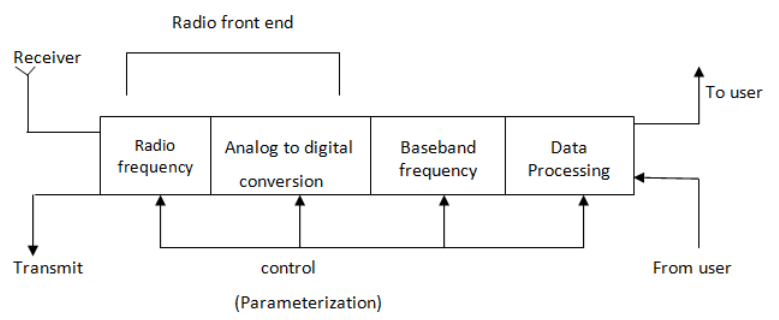

Fig 2: SDR transceiver

\section{SPECTRUM SENSING}

Spectrum sensing is the main element of the spectrum management. It emphasize on sensing the presence of primary user PU. Spectrum sensing is the first step to employ cognitive radio technology [5] [6]. It is a big challenge for spectrum sensing to find the unused band which is of similar frequency and operate its function on it. Spectrum sensing is done through a real- time wideband spectrum sensing ability to detect the presence of weak primary signals in a wide spectrum range. After the spectrum holes have been recognized then the cognitive radio user can operate them for their transmission. The process of finding the spectrum hole is called spectrum sensing. There are some issues like the multipath fading or shadowing of the PU signals, the changing levels of noise and signal to noise ratio (SNR), can significantly have an effect on the sensing accuracy which is discussed later in this paper.

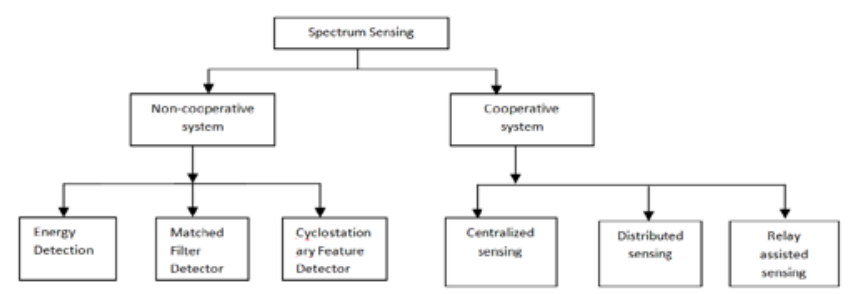

Fig3. Spectrum sensing techniques [7]

Generally there are two broad techniques of spectrum sensing under that broad techniques there are three narrow down techniques each which are used in spectrum sensing as shown in fig 3. In cooperative sensing all the decisions about sensing is taken mutually where as in non cooperative technique all the decisions is taken individually. [8][5]

(a) Need of spectrum sensing
In Today's are most of our spectrum is restricted by government organizations. The existing electromagnetic radio spectrum is partial resource and gets congested day by day that may result in signal problem or interference in connection, so to overcome this problem CR is introduced under which spectrum sensing is the essential component.[9] In wireless industry each wireless operator is assigned a particular licensed spectrum to operate its transmission in that frequency band only.CR technology is basically designed with a specific end goal to provide proper spectrum utilization and provide highly reliable communication.

\section{(b) Issues in spectrum sensing}

1. Channel uncertainty: The main cause of channel uncertainty is shadowing or fading under which it interpret mistakenly that primary user (PU) is present located out of the secondary users range but actually PU is experiencing fading. Hence CR must be more aware of the fact that which $\mathrm{PU}$ is faded and which is not.

2. Noise uncertainty: The detection performance can be improved by estimating noise power ratio. [10]

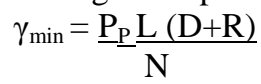

Where $\mathrm{N}$ is the noise power, $\mathrm{D}$ is the interface of the $\mathrm{SU}, \mathrm{P}_{\mathrm{p}}$ is the transmitted power of $\mathrm{PU}$ and $\mathrm{R}$ is the maximum distance between primary transmitter and corresponding receiver.

According to above equation in order to find out the required detection accuracy one must know the noise power which is not known in practical so it works on estimation by the receiver.

\section{MOBILE COMMUNICATION STANDARDS}

There are many standards used in wireless communication. [11]

(a) Bluetooth: It is a standard used for transmitting data over short distance. Bluetooth functions at frequencies between 2402 and $2480 \mathrm{MHz}$.It uses a radio technology called frequency-hopping spread spectrum. It split transmitted data into packets, and transmits each packet on one of 79 designated Bluetooth channels. Each channel has a bandwidth of $1 \mathrm{MHz}$.It usually performs 800 hops per second, with Adaptive Frequency-Hopping (AFH) enabled. [11]

(b) Cordless phone: It used DECT (Digital enhanced cordless telecommunication) to give cordless connection of receiver to fixed telephone system. Channel access mode of DECT is FDMA/TDMA.The transmission between connection is only secured by cyclic redundancy check(CRC). The product of bandwidth(B) and time $(\mathrm{T})$ is $\mathrm{BT}=0.5$.

(c) Wireless local area network: IEEE $802.11 \mathrm{~b}$ is generally used in Europe.IEEE 802.11a systems are also in development stage. If it is implemented in SDR, its modulation mode would be OFDM.

(d) Cellular System: GSM (Global system for mobile communication) is presently most successful standard for mobile communication. The product of bandwidth (B) and time $(\mathrm{T})$ is $\mathrm{BT}=0.3$.The transmission between connection is secured by CRC and convolution code, In Europe GSM system are operating in 900MHZ. 


\section{SPECTRUM SENSING IN CURRENT WIRELESS STANDARDS}

Recently many wireless standards started adopting cognitive features although it is very difficult to expect a use of underutilized spectrum in opportunistic manner in wireless standards.

(a) IEEE 802.11K: It is extension of IEEE 802.11. In this standard the sensing information is used to improve the traffic distribution. WLAN devices is connected to FC that has large signal level. In $802.11 \mathrm{k}$, when FC with large signal level is full of capacity then new users are allocated to one of the underutilized FC.

(b) Bluetooth: In Bluetooth standard, a new technology is introduced called AFW (Adaptive Frequency Hopping) to minimize interference between wireless technologies dividing 2.4GHZ unlicensed radio spectrum. In these IEEE 802.1.b/g devices, cordless phones and microwave ovens share the same wireless frequency with Bluetooth. There are many advantages of adopting AFH like clashing with WLAN signals are avoided, better bit error (BER) performance can be accomplished. AFH involves a sensing algorithm for determining whether ISM band has other devices present or to avoid them. The algorithm is based on information combined to decide which channel is occupied and which channel is vacant.

(c) IEEE 802.22: This standard is known as cognitive radio standard because it contains cognitive attributes. [12] This standard is still in growing stage. The most distinct feature of IEEE 802.22 is its spectrum sensing requirement. The sensing is predicted by two stages: Fast and Fine sensing, a crude sensing algorithm is used for example Energy detector. The fine sensing started with fast sensing results. Fine sensing involves more reliable sensing because more powerful tools or methods are used. All methods are discussed earlier in this paper.

\section{CONCLUSION}

Cognitive technology has been a promising and secured technique over many decades which facilitate the proper usage of spectrum as it is a very precious resource in wireless industry and it has been central point for research. In this paper, various standards used in cognitive radio have been reviewed. IEEE 802.22 is a standard used by cognitive radio. It encircles the SDR. The SDR stands on Digital Signal Processing and build on software with the adaptable
Radio Frequency components. Further different sensing issues have been discussed which is a big challenge itself in wireless industry

\section{REFERENCES}

[1]. I.F. Akyildiz, W.Y.Lee, M C. Vuran, S. Mohanty, "next generation/dynamic spectrum access/cognitive radio wireless networks: A survey”, computers networks, 50 (13), pp 21272159, 2006.

[2]. R.Garg, N.Saluja, "current trends and research challenges in spectrum sensing for cognitive radios", International journal of advanced computer science and applications, 7(2), pp 402408, 2016.

[3]. S.Singh, M.Kaur , "a survey on cognitive radio using spectrum sensing”, International journal of engineering research and general science , 3(2) , pp 1459- 1466, 2015.

[4]. I E. Igbinosa, O. Oyerinde, V M. Srivastava1, S.Mneney, "spectrum sensing methodologies for cognitive radio systems: a review”, International journal of advanced computer science and applications, 6(12), pp 13-22, 2015.

[5]. M D. Rana1, H R. Patel, "improving the existing spectrum sensing techniques for cognitive radio using modulation techniques", International journal of advanced research in computer and communication engineering, 3(9), pp 78727880, 2014.

[6]. I. F. Akyildiz, F.L Brandon, R. Balakrishnan, "cooperative spectrum sensing in cognitive radio networks: A survey" physical communication, 4(1) pp 40-62, 2011.

[7]. Shewangi, R.Garg, "review of cooperative sensing and non cooperative sensing in cognitive radio", International journal of engineering technology science and research, 4(5), pp 229234, 2017.

[8]. H.R Pous, M.J Blasco, "review of robust cooperative spectrum sensing techniques for cognitive radio networks" , wireless personal communication ,67(2) ,pp 175-198,2012.

[9]. K.Kumar, V.Sandhu, "comparison of non-cooperative spectrum sensing techniques in cognitive radio”, International journal of wired and wireless communications”, 4(1), pp 1518, 2015.

[10].M.Subhedar, G.Birajdar, "spectrum sensing techniques in cognitive radio networks: a survey”, International journal of next-generation networks, 3(2), pp 37-51, 2011.

[11].T.Yucek and H. Arslan, "A Survey of Spectrum Sensing Algorithms for Cognitive Radio Applications”, IEEE communications Surveys \& Tutorials, 11(1), pp. 116-130, 2009.

[12]. M.Nekovee, "a survey of cognitive radio access to tv white spaces”, International journal of digital multimedia broadcasting, Article ID 236568, pp 1-11, 2010. 